\title{
COMPARACIÓN DE ÍNDICES ANTROPOMÉTRICOS PARA AGREGACIÓN DE MÚLTIPLES FACTORES DE RIESGO EN ADULTOS DE CUENCA, ECUADOR
}

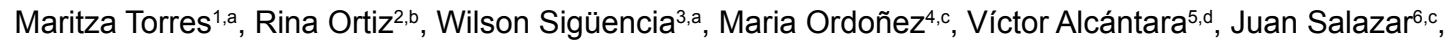 \\ Roberto Añez ${ }^{6, c}$, Joselyn Rojas ${ }^{7, e}$, Valmore Bermudez ${ }^{8, f}$
}

\begin{abstract}
RESUMEN
Objetivos. El objetivo de este estudio es comparar la capacidad predictiva de diferentes índices antropométricos en la determinación de la agregación de múltiples factores de riesgo (AMFR) en la población adulta de la ciudad de Cuenca, Ecuador. Materiales y métodos. Se realizó un estudio descriptivo transversal con un muestreo aleatorio multietápico en 318 sujetos adultos a quienes se les realizó una evaluación clínica, antropométrica y de laboratorio; siendo la circunferencia abdominal, índice de masa corporal (IMC) e índice cintura altura (ICA) los índices evaluados. La AMFR se definió como la presencia de $\geq$ dos componentes del síndrome metabólico (excluyendo circunferencia abdominal). Se realizaron curvas COR para determinar el área bajo la curva (ABC) para cada índice. Resultados. De los 318 individuos, un $54,1 \%(n=172)$ presentaron AMFR. Según los resultados obtenidos por curvas COR, la mayor capacidad predictiva en mujeres se observó con el IMC y el ICA (ABC: 0,751 y 0,750, respectivamente) mientras que en hombres la circunferencia abdominal y el ICA mostraron una capacidad predictiva similar $(A B C=0,762)$. El análisis multivariante ajustado por sexo y edad mostró que el ICA elevado (OR: 2,53; IC95\%: 1,12-5,71; $p=0,026$ ) fue el mejor predictor de AMFR, seguido por el IMC (OR: 2,15; IC95\%: $1,19-3,88 ; p=0,010)$. Conclusiones. La capacidad predictiva de los índices antropométricos está influenciada por el sexo, no obstante, el ICA es el mejor predictor de la AMFR en la población de Cuenca.
\end{abstract}

Palabras clave: Antropometría; Índices; Metabolismo; Obesidad; Curva ROC (fuente: DECS BIREME).

\section{COMPARISON OF ANTHROPOMETRIC MEASURES FOR AGGREGATION OF MULTIPLE RISK FACTORS IN ADULTS OF CUENCA, ECUADOR}

\begin{abstract}
Objective.The aim of this study is to compare the predictive capacity of different anthropometric indices in multiple risk factors aggregation (MRFA) determination in the adult population from Cuenca city, Ecuador. Materials and Methods. A crosssectional descriptive study was performed with a random multi-stage sampling in 318 adult subjects who underwent a clinical, anthropometric and laboratory evaluation; being the abdominal circumference, body mass index (BMI) and waist height index $(\mathrm{WH} \mathrm{tR}$ ) evaluated. MRFA was defined as the presence of $\geq 2$ components of the metabolic syndrome (excluding abdominal circumference). ROC curves were plotted to determine the area under the curve (AUC) for each index. Results. Of the 318 individuals, $54.1 \%(n=172)$ presented MRFA. According to ROC curves, the highest predictive capacity in women was observed with BMI and WHtR (AUC: 0.751 and 0.750 , respectively), while in men abdominal circumference and WHtR showed a similar predictive power (AUC: 0.762 ). The multivariate analysis adjusted for sex and age showed that high WHtR (OR: 2.53, 95\% Cl: 1.12-5.71, $\mathrm{p}=0.026$ ) was the best predictor of MRFA, followed by BMI (OR: $2.15,95 \% \mathrm{Cl}: 1.19-3.88, p=0.010$ ). Conclusions. The predictive capacity of the anthropometric indexes is influenced by gender; nevertheless the WHtR is the best predictor of MRFA in our population.
\end{abstract}

Keywords: Anthropometry; Indexes; Metabolism; Obesity; ROC curves (Source: MeSH NLM).

\footnotetext{
Centro de Salud de Baños, Ministerio de Salud Pública. Cuenca, Ecuador.

Universidad Católica de Cuenca. Facultad de Medicina. Cuenca, Ecuador.

Centro de Salud de San Pedro del Cebollar, Ministerio de Salud Pública. Cuenca, Ecuador.

Centro de Salud San Marcos, Ministerio de Salud Pública. Provincia de Santa Elena, Ecuador

Hospital El Ángel, Ministerio de Salud Pública. Cantón Espejo. Provincia del Carchi, Ecuador.

Centro de Investigaciones Endocrino Metabólicas «Dr. Félix Gómez». Facultad de Medicina. Universidad del Zulia. Venezuela.

Division of Pulmonary and Critical Car Medicine, Brigham and Women's Hospital and Harvard Medical School, Boston, EE.UU.

Facultad de Ciencias de la Salud, Universidad Simón Bolívar. Barranquilla, Colombia.

a Especialista en medicina familiar, magíster en endocrinologia avanzada; ' ${ }^{b}$ Especialista en docencia universitaria, magíster en endocrinologia avanzada; ${ }^{\mathrm{C}}$ Médico; ${ }^{\mathrm{d}}$ Médico Internista; ${ }^{\mathrm{e}}$ magíster en endocrinologia; ${ }^{\mathrm{f}}$ magíster en endocrinología, doctor en ciencias medicas

Recibido: 12/06/2017 Aprobado: 23/05/2018 En línea: 05/07/2018
}

Citar como: Torres M, Ortiz R, Sigüencia W, Ordoñez M, Alcántara V, Salazar J. et al. Comparación de índices antropométricos para agregación de múltiples factores de riesgo en adultos de Cuenca, Ecuador. Rev Peru Med Exp Salud Publica. 2018;35(2):198-204. doi:10.17843/rpmesp.2018.352.2938. 


\section{INTRODUCCIÓN}

En la actualidad los trastornos metabólicos constituyen los principales factores de riesgo para las enfermedades crónico degenerativas de mayor prevalencia a nivel mundial, siendo la obesidad la alteración más representativa y más relacionada a enfermedad cardiovascular (ECV), diabetes mellitus tipo 2, dislipidemia y síndrome metabólico (SM) (1); en los países en vías de desarrollo el uso de los parámetros antropométricos figura como una estrategia costo efectiva para definir la obesidad ante la falta de recursos para la utilización de mediciones directas del grado de adiposidad corporal (2). Dentro de estos parámetros los más empleados son el índice de masa corporal (IMC), la circunferencia abdominal y el índice cintura altura (ICA) por la facilidad de su determinación en la práctica clínica diaria y su alta sensibilidad en la predicción de eventos adversos relacionados a sobrepeso y obesidad ${ }^{(3)}$.

Pese a la simplicidad de su medición y de la importancia diagnóstica para el médico de atención primaria, en la región de Cuenca donde existen porcentajes tan elevados de obesidad ${ }^{(4)}$, los datos antropométricos no son comúnmente evaluados en el ejercicio diario en muchas consultas de atención primaria. Por lo tanto son necesarios estudios que demuestren la importancia de estos índices en la predicción de las enfermedades previamente mencionadas.

Entre los diferentes parámetros antropométricos, aún no existe un consenso acerca de la utilidad de uno sobre otro; en este sentido, a pesar de que en algunos reportes el IMC no parece distinguir entre los compartimientos corporales afectados al encontrarse elevado ${ }^{(5)}$, sigue siendo una de los más empleados por el personal de salud como método diagnóstico. Incluso un reporte previo en la ciudad de Cuenca demuestra que la circunferencia abdominal es un indicador antropométrico importante cuando se utilizan puntos de corte población específicos para definir obesidad abdominal ${ }^{(6)}$. En este sentido, dada la relevancia de la obesidad como factor de riesgo para un extenso número de enfermedades y el importante impacto que genera en materia de salud pública, determinar cuál de los índices antropométricos le ofrece al clínico una mayor predicción de eventos adversos es una potencial estrategia preventiva de abordaje en el adulto. Por ende, el objetivo de este estudio es comparar la capacidad predictiva de diferentes parámetros antropométricos en la determinación de la agregación de múltiples factores de riesgo en la población adulta de la ciudad de Cuenca, Ecuador.

\section{MATERIALES Y MÉTODOS}

\section{DISEÑO DEL ESTUDIO}

Estudio transversal descriptivo realizado en la ciudad de Cuenca, el cual utilizó valoraciones demográficas

\section{MENSAJES CLAVE}

Motivación para realizar el estudio. Siendo la obesidad un factor de riesgo para un extenso número de enfermedades de importancia en salud pública, es necesario determinar cuál de los índices antropométricos le ofrece al clínico una mayor predicción de eventos adversos.

Principales hallazgos. La mayor capacidad predictiva en mujeres se observó con el índice de masa corporal y la índice cintura altura mientras que en hombres fueron la medición de la circunferencia abdominal y del índice cintura altura. El índice cintura altura permite mayor capacidad predictiva y asociación con la agregación de múltiples factores de riesgo en la población adulta estudiada.

Implicancias. Los resultados demuestran la importancia de los diferentes parámetros antropométricos en la evaluación clínica de la obesidad y la necesidad de ser medidos en la práctica clínica habitual.

obtenidas del Instituto Nacional de Estadística y Censos (INEC censo 2010). La región urbana está dividida en las siguientes parroquias: San Sebastián, Bellavista, El Batán, Yanuncay, Sucre, Huayna Capac, Gil Ramírez Dávalos, Sagrario, San Blas, El Vecino, Cañaribamba, Totoracocha, Monay, Machangara, Hermano Miguel y El Resto con una población total de 329928 habitantes, se sumó a estos un grupo de 1960 individuos que habitan en parroquias rurales en el límite con las parroquias urbanas, haciendo un total de 331888 habitantes. Del total la población mayor de 18 años que habita el área urbana de la ciudad de Cuenca fue de 223798 individuos.

El cálculo del tamaño de la muestra para cada parroquia se realizó de forma proporcional mediante la fórmula de Sierra Bravo la cual emplea la prevalencia de la enfermedad a analizar, el total de sujetos calculados para cada parroquia (promedio: 19) fue sumado, obteniéndose una muestra final de 318 individuos. El muestreo realizado fue de tipo aleatorio multietápico por conglomerados, estos conglomerados estuvieron representados de forma descendente por parroquias, sectores, manzanas y casas (unidad básica o mínima de muestreo). Posteriormente mediante un método aleatorio, todas las personas con edad $\geq 18$ años de cada casa fueron sorteadas, seleccionándose un participante para el estudio durante el periodo octubre 2013 a febrero de 2014. El criterio de inclusión fue todos los habitantes mayores de 18 años sorteados aleatoriamente, dentro de los criterios de exclusión se consideraron mujeres en periodo de gestación, individuos recluidos en instituciones penales, hospitales, o cuarteles militares.

Este reporte representa un sub-análisis (análisis secundario) donde se evaluaron específicamente los parámetros antropométricos de los 318 sujetos, en el estudio primario se determinó el punto de corte de circunferencia abdominal en la ciudad de Cuenca ${ }^{(6)}$, tal como lo recomendó el consenso de SM 2009. 


\section{EVALUACIÓN DE LOS INDIVIDUOS}

Tras la selección de los sujetos a evaluar, estos fueron citados a una entrevista en el centro de salud más cercano a su hogar donde se les realizó una historia clínica completa, obteniéndose datos y antecedentes personales.

\section{EVALUACIÓN ANTROPOMÉTRICA}

El peso fue medido con la balanza Camry de plataforma y pantalla grande tipo aguja de reloj, modelo DT602, capacidad $130 \mathrm{~kg}$, de piso con alfombrilla, país de origen China; la talla fue evaluada con tallímetro, marca Seca 217 , país de origen Alemania; la circunferencia abdominal con una cinta métrica a la altura de la línea media axilar en el punto imaginario que se encuentra entre la parte inferior de la última costilla y el punto más alto de la cresta ilíaca, en posición de pies, al final de una espiración ${ }^{(7)}$. El ICA se determinó al dividir la circunferencia abdominal $(\mathrm{cm})$ entre la talla $(\mathrm{cm})$. El índice de masa corporal se calculó utilizando la fórmula (peso/talla ${ }^{2}$, expresada en $\left.\mathrm{kg} / \mathrm{m}^{2}\right)^{(8)}$.

\section{ANÁLISIS DE LABORATORIO}

La extracción de sangre se realizó tras un periodo de ayuno de ocho a 12 horas y en las primeras horas de la mañana, el personal del laboratorio "Paucarbamba» de la ciudad de Cuenca analizó las muestras; para lo cual se le extrajo a cada individuo cinco $\mathrm{cm}^{3}$ de sangre obtenida por venopunción antecubital, colocándose en tubos Vacutainer. Se determinaron niveles de colesterol total, triglicéridos (TAG), colesterol de alta y baja densidad (HDL-C, LDL-C respectivamente) y glucosa plasmática. Mediante el equipo Mindray b 88 semiaulan; se determinó la concentración de glicemia, colesterol total y TAG, para la cuantificación de LDL-C se utilizó un kit enzimático-colorimétrico de (reaactlab), para HDL-C se utilizó un kit enzimáticocolorimétrico comercial (Human Frisonex).

\section{DEFINICIONES}

La Agregación de Múltiples Factores de Riesgo (AMFR) fue definida como la presencia de $\geq$ dos factores de riesgo cardiometabólico ${ }^{(9)}$, empleándose las variables: TAG elevados: $\geq 150 \mathrm{mg} / \mathrm{dl}$, HDL-C bajas: (Hombres $<40 \mathrm{mg} /$ dl), (Mujeres $<50 \mathrm{mg} / \mathrm{dl}$ ), presión arterial elevada: $\geq 130 / 85$ $\mathrm{mmHg}$ o diagnóstico previo de hipertensión arterial, glicemia elevada: $\geq 100 \mathrm{mg} / \mathrm{dl}$ o diagnóstico previo de DM2, o el uso de medicamentos para el control de cada alteración respectiva.

\section{ANÁLISIS ESTADÍSTICO}

Las variables cualitativas fueron presentadas como frecuencias absolutas y relativas (porcentaje), se utilizó la prueba de chi cuadrado $\left(X^{2}\right)$ para determinar la asociación entre variables cualitativas. Para evaluar la distribución normal de las variables cuantitativas se utilizó la prueba de
Kolmogorov Smirnov, las variables con distribución normal, fueron expresadas con media \pm desviación estándar. Las curvas COR se realizaron para determinar el área bajo la curva $(A B C)$ de cada parámetro antropométrico, para la selección del puntos de corte se empleó el índice de Youden, la distancia al punto más cercano a 0,1 y la comparación entre $A B C$ se realizó mediante la prueba Delong. Adicionalmente, se realizó un modelo de regresión logística para AMFR ajustado por: sexo, grupos etarios y cada uno de los índices definidos por los puntos de corte obtenidos durante el análisis (circunferencia abdominal: mujeres: > 91,5cm; hombres: > 95,5cm; ICA: mujeres: $\geq 0,59$; hombres: $\geq 0,59$; IMC: $\geq 26 \mathrm{Kg} / \mathrm{m}^{2}$ en ambos sexos). Los datos fueron analizados mediante el paquete informático para Ciencias Sociales SPSS versión 20, para Windows (SPSS Inc. Chicago, IL), se consideraron los resultados estadísticamente significativos cuando $\mathrm{p}<0,05$.

\section{ASPECTOS ÉTICOS}

Los individuos que participaron en el estudio firmaron un consentimiento informado en el cual se expuso el respeto a la vida, la salud, la confidencialidad, la intimidad y la dignidad, asimismo se les explicó todos los detalles concernientes al estudio y los procedimientos a los cuales iban a ser sometidos, antes de realizarles el examen clínico, físico y de laboratorio. El estudio fue aprobado por el comité de ética del Centro de Salud de Baños y Centro de Salud de San Pedro del Cebollar, cantón Cuenca, provincia del Azuay, Distrito de salud 01D02 perteneciente al Ministerio de Salud Pública del Ecuador.

\section{RESULTADOS}

\section{CARACTERÍSTICAS DE LA POBLACIÓN ESTUDIADA}

La muestra estuvo conformada por 318 individuos de los cuales el $57,2 \%$ ( $n=182)$ fueron mujeres. La edad promedio fue de 42,8 $\pm 15,5$ años. El grupo etario más frecuente fue el de menos de 40 años con 44,7\% ( $n=142)$, la agregación de múltiples factores de riesgo estuvo presente en $54,1 \%$ $(n=172)$ de los sujetos (Tabla 1).

\section{CURVAS COR Y PUNTOS DE CORTE DE ÍNDICES ANTROPOMÉTRICOS}

En la Figura 1 se muestran las curvas COR de cada parámetro antropométrico para AMFR según sexo. En la Tabla 2 se observan los puntos de corte seleccionados para cada parámetro con sus respectivos índices. Para circunferencia abdominal los puntos de corte fueron 91,5 $\mathrm{cm}$ para mujeres [ABC: $0,730(0,656-0,803)$; sensibilidad: $66,7 \%$ y especificidad: $62,7 \%$ ] y $95,5 \mathrm{~cm}$ para hombres [ABC: $0,762(0,683-0,840)$; sensibilidad: $71,2 \%$ y especificidad: $68,3 \%$. En cuanto al ICA el punto de corte para mujeres fue 0,59 [ABC: 0,750 (0,678-0,822); sensibilidad: $72,7 \%$ 
Tabla 1. Características generales de personas mayores de 18 años de la ciudad de Cuenca, Ecuador

\begin{tabular}{|c|c|c|c|c|c|c|}
\hline & \multicolumn{2}{|c|}{ Femenino } & \multicolumn{2}{|c|}{ Masculino } & \multicolumn{2}{|c|}{ Total } \\
\hline & $n=182$ & $\%$ & $n=136$ & $\%$ & $n=318$ & $\%$ \\
\hline \multicolumn{7}{|l|}{ Grupos etarios (años) } \\
\hline$<40$ & 84 & 46,2 & 58 & 42,6 & 142 & 44,7 \\
\hline $40-59$ & 68 & 37,4 & 56 & 41,2 & 124 & 39,0 \\
\hline$\geq 60$ & 30 & 16,5 & 22 & 16,2 & 52 & 16,4 \\
\hline Triglicéridos elevadosł & 70 & 38,5 & 62 & 45,6 & 132 & 41,5 \\
\hline HDL-C bajoł & 142 & 78,0 & 78 & 57,4 & 220 & 69,2 \\
\hline Presión arterial elevadał & 63 & 34,6 & 61 & 44,9 & 124 & 39,0 \\
\hline Hiperglicemiał & 33 & 18,1 & 25 & 18,4 & 58 & 18,2 \\
\hline Agregación de múltiples factores de riesgo† & 99 & 54,4 & 73 & 53,7 & 172 & 54,1 \\
\hline
\end{tabular}

‡ Según los criterios del Consenso IDF/AHA/NHLBI/IASO/WHF 2009

† Presencia de $\geq 2$ criterios de Síndrome Metabólico (excluyendo circunferencia abdominal)

y especificidad: $67,5 \%]$ y para hombres fue 0,57 [ABC: $0,762(0,683-0,840)$; sensibilidad: $71,2 \%$ y especificidad: $65,1 \%$. Para IMC el punto de corte para mujeres fue 26,3 $\mathrm{Kg} / \mathrm{m}^{2}$ [ABC: 0,751 (0,681-0,822); sensibilidad: $70,7 \%$ y especificidad: $67,5 \%$ ] y para hombres fue $26,2 \mathrm{Kg} / \mathrm{m}^{2}$ [ABC: $0,717(0,632-0,802)$; sensibilidad: $67,1 \%$ y especificidad: $60,3 \%$. Las comparaciones entre ABC para cada índice se observan en la Tabla 3.

\section{PARÁMETROS ANTROPOMÉTRICOS Y AGREGACIÓN DE MÚLTIPLES FACTORES DE RIESGO}

Al evaluar la distribución de individuos según los parámetros analizados y su relación con la AMFR se observa que aquellos con ICA elevada presentan un mayor porcentaje de afectados (ICA elevada: sin AMFR: $37,0 \%$ vs con AMFR: 76,2\%; $x^{2}=49,81 ; p<0,0001$; OR: 2,53 IC95\%: 1,12-5,71; $p=0,026)$. Seguido del IMC y la circunferencia abdominal en cuanto a importancia (Tabla 4).

\section{DISCUSIÓN}

Los hallazgos muestran que, en el sexo femenino, tanto el ICA como el IMC presentan la mayor capacidad predictiva para la AMFR, similar a los planteados por Mora-García y cols. ${ }^{(10)}$, quienes evidenciaron que el ICA fue el mejor predictor de SM en 434 mujeres de Cartagena, Colombia. Asimismo, Bermúdez y cols. ${ }^{(11)}$, también plantean este índice como el más importante en la población femenina de Maracaibo, Venezuela. Por su parte, en el sexo masculino

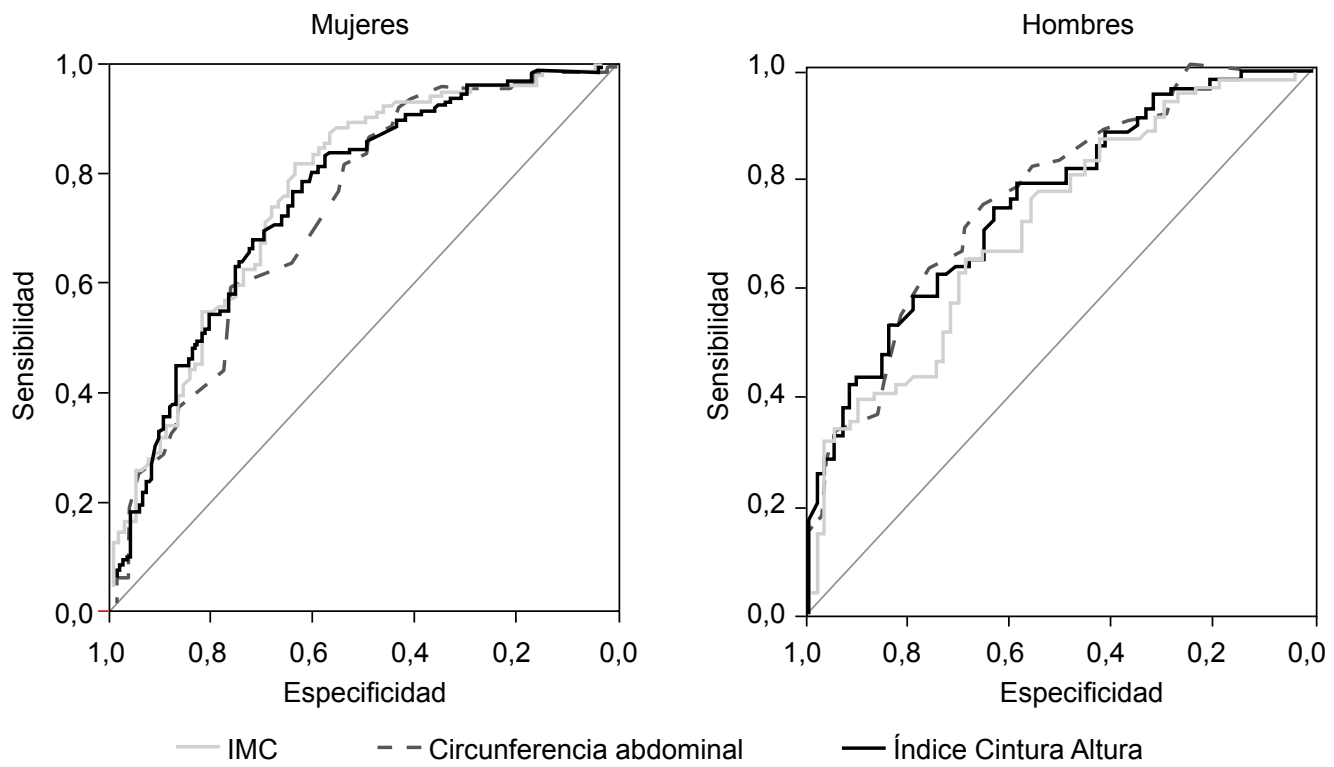

Figura 1. Curvas COR de índices antropométricos para la agregación de múltiples factores de riesgo. Cuenca, Ecuador 
Tabla 2. Puntos de corte de índices antropométricos en personas mayores de 18 años de la ciudad de Cuenca, Ecuador

\begin{tabular}{lccc}
\hline & & Índices Antropométricos & \\
\cline { 2 - 4 } & Circunferencia Abdominal & Índice Cintura-Altura & Índice de Masa Corporal \\
\hline Femenino & & & \\
Punto de Corte & $91,5 \mathrm{~cm}$ & 0,59 & $26,3 \mathrm{Kg} / \mathrm{m}^{2}$ \\
ABC (Cl 95\%) & $0,730(0,656-0,803)$ & $0,750(0,678-0,822)$ & $0,751(0,681-0,822)$ \\
Sensibilidad (\%) & 66,7 & 72,7 & 70,7 \\
Especificidad (\%) & 62,7 & 67,5 & 67,5 \\
Índice Youden & 0,29 & 0,40 & 0,38 \\
Distancia ROC & 0,500 & 0,424 & 0,437 \\
Masculino & & & $26,2 \mathrm{Kg} / \mathrm{m}^{2}$ \\
Punto de Corte & $95,5 \mathrm{~cm}$ & 0,57 & $0,717(0,632-0,802)$ \\
ABC (CI 95\%) & $0,762(0,683-0,840)$ & $0,762(0,683-0,840)$ & 67,1 \\
Sensibilidad (\%) & 71,2 & 71,2 & 60,3 \\
Especificidad (\%) & 68,3 & 65,1 & 0,27 \\
Índice Youden & 0,40 & 0,36 & 0,515 \\
\hline Distancia ROC & 0,428 & 0,452 & \\
\hline
\end{tabular}

la circunferencia abdominal y el ICA fueron los mejores predictores encontrados en la población analizada, similar a los resultados obtenidos en la ciudad de Maracaibo y a los exhibidos en 1552 sujetos de Qatar donde la circunferencia abdominal representó el mejor parámetro antropométrico predictor de $\mathrm{SM}^{(12)}$

No obstante, es importante considerar la diferencia en la utilización de la AMFR y el diagnóstico de SM como variable objetivo o final, en esta última la circunferencia abdominal forma parte de los componentes del síndrome (y a su vez es uno de los parámetros antropométricos analizados o incluidos en los índices). Al respecto, coincidimos con el planteamiento de Bermúdez y cols., sobre la necesidad de excluir las medidas antropométricas en las variables finales a calcular dada la posible autocorrelación que puede producirse durante el análisis estadístico, generando sesgos en la interpretación (11).

Es interesante observar como el IMC fue inferior a la circunferencia abdominal en la predicción de AMFR sólo en el sexo masculino, concordante con los resultados de
Beydoun y cols. ${ }^{(13)}$, en 1981 sujetos del estudio HANDLS; lo cual puede estar relacionado a la influencia de la masa muscular como un factor de confusión durante el cálculo del peso en hombres.

Otro hallazgo a resaltar en relación al IMC es el punto de corte a partir del cual se predice mejor la presencia de AMFR, el cual es muy inferior al empleado para definir obesidad según la Organización Mundial de la Salud (OMS). Estas diferencias ya evidenciadas y aplicadas en las poblaciones asiáticas desde hace años ${ }^{(14)}$, demuestran la necesidad de evaluar en futuros estudios si realmente los puntos de corte empleados para definir obesidad en la población latinoamericana son los suficientemente sensibles para identificar a todos los sujetos enfermos, considerando la heterogeneidad de esta enfermedad y la presencia de fenotipos metabólicos atípicos, difíciles de distinguir de forma rutinaria en la práctica clínica ${ }^{(15)}$.

Un resultado constante en nuestro estudio es la capacidad predictiva del ICA, el cual hasta hace algunos años era evaluado exclusivamente en la población infantil (16). En

Tabla 3. Comparaciones de área bajo la curva para cada índice antropométrico según sexo en personas mayores de 18 años de la ciudad de Cuenca, Ecuador

\begin{tabular}{lcc}
\hline Comparación & ABC & Prueba Delong ( $\boldsymbol{p})$ \\
\hline Femenino & & \\
$\quad$ Circunferencia abdominal - Índice de masa corporal & $0,730-0,751$ & 0,341 \\
$\quad$ Circunferencia abdominal - Índice cintura altura & $0,730-0,750$ & 0,682 \\
$\quad$ Índice de masa corporal - Índice cintura altura & $0,751-0,750$ & 0,493 \\
Masculino & & $1,99 \times 10^{-7}$ \\
$\quad$ Circunferencia abdominal - Índice de masa corporal & $0,762-0,717$ & 0,602 \\
$\quad$ Circunferencia abdominal - Índice cintura altura & $0,762-0,762$ & $2,98 \times 10^{-6}$ \\
\hline Índice de masa corporal - Índice cintura altura & $0,717-0,762$ & \\
\hline
\end{tabular}

$A B C$ : área bajo la curva 
Tabla 4. Relación entre índices antropométricos y agregación de múltiples factores de riesgo en personas mayores de 18 años de la ciudad de Cuenca, Ecuador

\begin{tabular}{|c|c|c|c|c|c|c|c|}
\hline & & & & & & & \\
\hline & $n=146$ & $\%$ & $n=172$ & $\%$ & Valor de $p$ & OR (IC95\%) & Valor de $\mathrm{p}^{*}$ \\
\hline Circunferencia Abdominalł & & & & & $<0,001$ & & \\
\hline Normal & 95 & 65,1 & 54 & 31,4 & & 1,00 & \\
\hline Elevada & 51 & 34,9 & 118 & 68,6 & & $0,99(0,45-2,18)$ & 0,989 \\
\hline 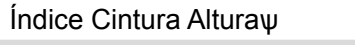 & & & & & $<0,001$ & & \\
\hline Normal & 92 & 63,0 & 41 & 23,8 & & 1,00 & \\
\hline Elevada & 54 & 37,0 & 131 & 76,2 & & $2,53(1,12-5,71)$ & 0,026 \\
\hline Índice de Masa Corporalף & & & & & $<0,001$ & & \\
\hline Normal & 91 & 62,3 & 49 & 28,5 & & 1,00 & \\
\hline Elevado & 55 & 37,7 & 123 & 71,5 & & $2,15(1,19-3,88)$ & 0,010 \\
\hline
\end{tabular}

*Modelo ajustado por: sexo, grupos etarios, grupos étnicos, y cada uno de los índices definidos por los puntos de corte obtenidos.

AMFR=Agregación de múltiples factores de riesgo

$\ddagger>91,5 \mathrm{~cm}$ (mujeres) y $>95,5 \mathrm{~cm}$ (hombres)

$\psi \geq 0,59$ (mujeres) y $\geq 0,57$ (hombres)

II $\geq 26 \mathrm{Kg} / \mathrm{m}^{2}$ en ambos sexos

la actualidad, diversos estudios a gran escala e incluso meta-análisis han demostrado su utilidad en la población adulta ${ }^{(17)}$, aún cuando los valores de IMC y circunferencia abdominal se encuentran normales ${ }^{(18)}$. Los puntos de corte obtenidos para mujeres y hombres, fueron 0,59 y 0,57 , respectivamente; ligeramente superiores al planteado por Li y cols. ${ }^{(19)}$, para la población taiwanesa en un estudio transversal en más de 30000 individuos. Al evaluar desde el punto de vista multivariante la relación de cada parámetro, se muestra que aquellos sujetos con ICA elevado tienen hasta 2,5 veces más riesgo de presentar la AMFR, superior al riesgo mostrado por IMC y circunferencia abdominal, este último siendo no estadísticamente significativo.

En conclusión, los resultados demuestran la importancia de los diferentes parámetros antropométricos en la evaluación clínica de la obesidad y la necesidad de ser medidos en la práctica clínica habitual dada su costo efectividad. El ICA es el índice con mayor capacidad predictiva y mayor asociación con la AMFR en la población adulta de la ciudad de Cuenca. Pese al carácter transversal del estudio, lo cual no permite establecer causalidad en la relación encontrada, este representa el inicio de una serie de análisis de la influencia de las medidas antropométricas sobre los diferentes factores de riesgo cardiovascular. Asimismo, la categorización de las variables antropométricas representa otra limitación, dada la mediana correlación obtenida en el análisis cuantitativo, una práctica que es común en los estudios poblacionales que valoran estos índices.

Contribuciones de autoría: MT participó en la concepción y diseño del artículo y recolección de resultados, RO participó en la concepción, diseño del artículo y redacción del artículo, WS participó en la recolección de resultados y redacción del artículo, MO y VA participaron en el aporte aporte de pacientes o material de estudio y asesoría técnica, administrativa. RA y JS participaron en el análisis e interpretación de datos, asesoría estadística, JR participó en el análisis e interpretación de datos y revisión crítica del artículo, VB participó en la concepción y diseño del artículo y revisión crítica del artículo. Todos los autores aprobaron la versión final

Financiamiento. Autofinanciado.

Conflicto de intereses: Los autores declaran no tener conflictos en la publicación de este artículo.

\section{REFERENCIAS BIBLIOGRÁFICAS}

1. Kaur J. A Comprehensive Review on Metabolic Syndrome. Cardiol Res Pract. 2014; 2014: 943162.

2. Mony PK, Swaminathan S, Gajendran JK, Vaz M. Quality Assurance for Accuracy of Anthropometric Measurements in Clinical and Epidemiological Studies: [Errare humanum est $=$ to err is human]. Indian J Community Med. 2016; 41(2): 98-102.

3. Kee CC, Jamaiyah H, Geeta A, Ali ZA, Safiza MN, Suzana S, Khor GL, Rahmah
R, Jamalludin AR, Sumarni MG, Lim KH, Faudzi YA, Amal NM. Sensitivity and specificity of waist circumference as a single screening tool for identification of overweight and obesity among Malaysian adults. Med J Malaysia. 2011;66(5):462-7.

4. Vásquez MA, et al. Prevalencia y nivel de concordancia entre tres definiciones de síndrome metabólico en la ciudad de cuenca-Ecuador. Avances en Biomedicina. 2016;5(3):117-128.
5. Shah NR, Braverman ER. Measuring Adiposity in Patients: The Utility of Body Mass Index (BMI), Percent Body Fat, and Leptin. PLoS One. 2012; 7(4): e33308.

6. Torres M, Ortiz R, Sigüenza W, et al. Punto de corte de circunferencia abdominal para el agrupamiento de factores de riesgo metabólico: una propuesta para la población adulta de Cuenca, Ecuador. Rev Argent Endocrinol Metab. 2016;53(2):59-66.

7. Statistics. NHANES III reference manuals and reports (CDROM). Hyattsville, 
MD: Centers for Disease Control and Prevention, 1996. Citado: 12/04/17. Disponible en: http://www.cdc.gov/nchs/ data/nhanes/nhanes3/cdrom/nchs/manuals/anthro.pdf

8. Sámano R, Zelonka R, Martínez-Rojano $\mathrm{H}$, Sánchez-Jiménez $\mathrm{B}$, Ramírez $\mathrm{C}$, Ovando G. Asociación del índice de masa corporal y conductas de riesgo en el desarrollo de trastornos de la conducta alimentaria en adolescentes mexicanos. Arch Latinoam Nutr. 2012;62(2):145-54.

9. Mora-García GJ, Gómez-Camargo D, Mazenett E, Alario Á, Fortich Á, GómezAlegría C. Anthropometric parameters' cut-off points and predictive value for metabolic syndrome in women from Cartagena, Colombia. Salud Publica Mex. 2014;56(2):146-53.

10. Mora-García GJ, Gómez-Camargo D, Mazenett E, Alario Á, Fortich Á, GómezAlegría C. Anthropometric parameters' cut-off points and predictive value for metabolic syndrome in women from Cartagena, Colombia. Salud Publica Mex. 2014;56(2):146-53.

11. Bermúdez VJ, Rojas J, Salazar J, Añez R, Martínez MS, et al. Anthropometric Predictors for Multiple Risk Factor
Aggregation in Adults from Maracaibo City.J Obes Overweig 2015;1(3): 301.

12. Bener A, Yousafzai MT, Darwish S, Al-Hamaq AO, Nasralla EA, Abdul-Ghani M. Obesity index that better predict metabolic syndrome: body mass index, waist circumference, waist hip ratio, or waist height ratio. J Obes. 2013;2013:269038.

13. Beydoun MA, Kuczmarski MT, Wang Y, Mason MA, Evans MK, Zonderman AB. Receiver-operating characteristics of adiposity for metabolic syndrome: the Healthy Aging in Neighborhoods of Diversity across the Life Span (HANDLS) study. Public Health Nutr. 2011;14(1):77-92.

14. Misra A. Ethnic-Specific Criteria for Classification of Body Mass Index: A Perspective for Asian Indians and American Diabetes Association Position Statement. Diabetes Technol Ther. 2015; 17(9): 667-671.

15. Blüher M. Are metabolically healthy obese individuals really healthy? Eur J Endocrinol. 2014;171(6):R209-19.

16. McCarthy HD, Ashwell M. A study of central fatness using waist-to-height ratios in UK children and adolescents over two decades supports the simple message-'keep your waist circumference to less than half your height.' Int J Obes (Lond) 2006;30:988-92.

17. Savva SC, Lamnisos D, Kafatos AG. Predicting cardiometabolic risk: waist-toheight ratio or BMI. A meta-analysis. Diabetes Metab Syndr Obes. 2013;6:403-19.

18. Zhu Q, Shen F, Ye T, Zhou Q, Deng H, Gu $\mathrm{X}$. Waist-to-height ratio is an appropriate index for identifying cardiometabolic risk in Chinese individuals with normal body mass index and waist circumference. J Diabetes. 2014;6(6):527-34.

19. Li WC, Chen IC, Chang YC, Loke SS, Wang SH, Hsiao KY. Waist-to-height ratio, waist circumference, and body mass index as indices of cardiometabolic risk among 36,642 Taiwanese adults. Eur J Nutr. 2013;52(1):57-65.

Correspondencia: Maritza Torres Dirección: Centro de Salud de Baños. Ciudad de Cuenca, Provincia del Azuay, República del Ecuador. Teléfono: 0986511936: (07) 22855721

Correo electrónico: Torres.maritza1978@gmail.com 